general principles underlying the procedures used, drawing on the extensive experience of the New York group using insulin, growth hormone, ACTH and parathyroid hormone. Requirements of the labelled hormones were regarded as less stringent than is widely supposed, the essential one being that the plasma hormone and a standard preparation should be equivalent in their capacity to inhibit the reaction between labelled hormone and antiserum. It follows that neither the animal species from which the standard preparation, or the preparation for iodination, is obtained nor, within limits, the degree of damage suffered during iodination is critical. There was general agreement, however, that some purification of the labelled hormone is essential and methods for doing this were described. The possibility that metabolic fragments of endogenous hormones which are immunologically but not hormonally active might be present in plasma was also discussed. The general problem of damage to hormones was included in a round table session organized by Milhaud (Paris).

Some fifteen successful assays using the chloramine $T$ method have been carried out in various laboratories, but Greenwood (London) referred to unsatisfactory experiences with iodine-131 obtained since 1964. Results with the method in the United States had been more consistent than those obtained elsewhere and this seemed to depend mainly on higher specific activities of the available iodine-131. Michel (Paris), Quabbe (Berlin), Brauman (Brussels) and Franchimont (Liège) had broadly shared Greenwood's experience. The determination of ${ }^{131} \mathrm{I}$ specific activities was outlined by Yalow. In view of the lack of knowledge of iodine and radioiodine chemistry emphasized by Hughes any improvement in the precision of micro-iodine estimations must be welcomed.

The availability of standard preparations of human growth hormone and antisera was discussed by various speakers including Coates (London) and Stewart (Frankfurt). Rosa (Pisa) and Ooms (Brussels) presented short communications on the immunological and biological properties of radioiodinated insulins, while Melani (Frankfurt) reported a procedure for assaying adrenocorticotrophic hormone (ACTH) which depends on the use of an ion exchange resin. The sensitivity of his assay was equivalent to that of the first ACTH one reported by Berson et al., and this has since been greatly increased. Crosignani and Polvani (Milan) and Freedlender (Boston) reported on assays for human chorionic gonadotrophin and growth hormone respectively which differed in detail from previous assays using the double antibody method. Franchimont (Liège) outlined assays both for human follicle-stimulating hormone and luteinizing hormone, and reported millimicrogram levels during the normal menstrual cycle with two peaks of luteinizing hormone and one of follicle-stimulating hormone. A full report of his procedure is awaited with considerable interest.

In 1963 groups in New York and Washington jointly reported an assay for parathyroid hormone, the procedure having since been improved in sensitivity and applied to humans and animals. Yalow summarized the present position of the New York group and gave unpublished results of human plasma measurements from which it was evident that elinical application of the method is still hindered by its lack of sensitivity. Potts, on behalf of the Washington group, showed data bearing on the control of animal secretion of the hormone. In the course of this. work an identical reaction between crude human parathyroid hormone and purified bovine parathyroid hormone was demonstrated. Availability of purified bovine hormone and antiserum was also discussed.

The clinical and physiological applications of radioimmunoassays were only outlined for insulin by Semols (London) and for glucagon by Weinges (Homburg-Saar) representing Unger's group. Both communications served inter alia to emphasize the usefulness of procedures which can be carried out at rates of up to 200 measurements per week. A discrepancy was noted in the levels of glucagon found in plasma by Samols and Unger, and this may explain the differences in their results after oral administration of glucose.

Abstracts of the papers presented are available $(J$. Labelled Comp., 1, No. 4, 311) and a full report in book form will appear in due course.

A. S. McFarLane

F. C. Greenwood

\title{
DOMESTIC FOOD CONSUMPTION AND EXPENDITURE
}

$D^{o}$ MESTIC Food Consumption and Expenditure, 1963, is the fourteenth annual report produced by the National Food Survey Committee*. The 1962 report reviewed the findings of surveys conducted during the years 1950-60. Over such a period it is easier to observe changing trends in consumption patterns and the quantities of food consumed or purchased than from one year to the next. Nevertheless, interesting facts do emerge from the present report.

Survey methods remained unchanged, but for reasons of economy the sample in the 1963 survey was reduced by 18 per cent as compared with the 1962 sample, tho effective size being 7,532 households. There was no change in the average number of persons per household, which again was least in London $(2.95)$ and highest in rural areas (3.41). Because of random sampling, rural areas were over-represented in the 1963 survey, and in order to correct this bias the national averages in this report have been obtained by weighting the separate averages for the different types of urban and rural areas by the estimated population resident in those areas. The composition of the population is interesting. In social class $A$ there were only eight households with four or

- Ministry of Agrieulture, Fisheries and Food. Domestic Food Consumption and Expenditure. 1963-Annual Report of the National Food Survey (ommittee. Pp. $\mathrm{ix}+131$. (London: H.M.S.O., 1965.) 128. 6d. net. more children and only very cautious conclusions can be drawn from this sample. The representation of old-age pensioners, of whom two-thirds were women, further increased. It appears that the highest proportion of elderly men live in the south-west region. The average number of earners per household tended to vary inversely with the income of heads of households including a number of children, and adult earners tended to be fewer in Class $D$ than in any other earning classes because of the smaller average number of adults per household. The highest number of earners was in families containing adolescents.

The exceptional severity of the 1963 winter adversely affected the supply and distribution of certain foods and this in turn affected prices during the first quarter of the year. Over the year, and for the country as a whole, the average total expenditure was $32 s$. $4 d$. per week, a slight increase over 1962, which can be explained partly by a rise in food prices and partly by an increase in the quantity of food purchases. Expenditure on 'convenience foods' rose to $6 s .1 d$. per head per week as compared with $5 s .11 d$. in 1962, but remained at about 19 per cent of the household food budget. Consumption of dairy products and eggs increased, while the consumption of fish fell. Consumption of poultry increased, though at a slower rate than previously, probably at the expense of meat, the 
consumption of which showed a slight decrease. The demand for tea remains inelastic, in spite of changes in price or income, but the demand for coffee has risen, particularly for 'instant coffee', purchases of which were nearly 80 per cent higher than in 1960.

The tables showing the expenditure on and consumption of food are informative. Expenditure per week was highest $(50 s .3 d$.$) in closs A$ for families containing two adults under 55 , falling to $27 s$. $4 d$. for a family with three children; it was lowest in classes $C$ and $D$, where $19 s .9 d$. was spent by the family with four or more children. The lowest consumption per head per week of a high proportion of foods was most frequently recorded in families with four or more children; for bread, vegetables and beverages, such as tea, however, families with two or three children occupied this position. For all foods except tea taken by families of two adults over 55, the highest consumers consisted of families of two adults under 55. With respect to differences in social classes, class $A$ were, as might be expected, the highest consumers of the more expensive foods such as dairy produce, eggs, meat, chicken, fish and coffee, whereas old-age pensioners led with tea and sugar, and class $C$ with vegetables. The cheaper foods, bread and cereals, sugar and tea, were taken in the smallest quantity by class $A$, whereas classes $C$ and $D$ consumed less of all the other food groups, being joined by the oldage pensioners in the low consumption of meat and fruit.

The energy value of the diet of the average person remains much the same as in 1962 , with class $A$ and oldage pensioners having the highest intake of calories. The percentage of calories provided by protein remains unchanged at 11.5 per cent. The level of intake of other nutrients in families appeared satisfactory when expressed as a percentage of recommended requirements, but this may not apply to all individuals. The way in which food is distributed within the family is unknown, and possibly some members of some families were abundantly fed at the expense of others.
Margaret E. CaMeron

\title{
ABUNDANCE ANALYSIS OF THE PECULIAR STAR HD 101065
}

\author{
By DR. A. PRZYBYLSKI \\ Mount Stromlo Observatory, Australian National University, Canberra
}

$\mathrm{T}$

HE eighth magnitude star HD 101065 ( $\alpha=11 \mathrm{~h} 35 \cdot 2 \mathrm{~m}$, $\delta=-46^{\circ} 26^{\prime}, 1950 \cdot 0$ ) has a unique and extremely peculiar spectrum ${ }^{1-4}$. While spectral peculiarities are superimposed on a more or less normal spectrum in other abnormal stars, there are scarcely any normal features in the spectrum of this star. Most striking is the absence of the elements usually seen in the spectra of normal stars such as iron, titanium, chromium, manganese and sodium, and the great strength of lines of barium and the rare earths. Hydrogen, calcium, barium and possibly strontium and lithium are the only elements which can be seen in the spectrum besides the ionized rare earths. The hydrogen lines are of normal width but they are much shallower than in a normal star of similar temperature. The calcium $H$ and $K$ lines are sharp and are weaker than many strong lines of the rare earths. The $K$ line, with its equivalent width of $0.3 \AA$, is about sixty times weaker than in the solar spectrum even though we are dealing with a star of similar temperature.

Counting each local minimum of intensity as a separate line, about 3,000 lines have been measured between $\lambda 3600$ and $\lambda 4950 \AA$ on plates with a dispersion of $6 \cdot 7 \AA / \mathrm{mm}$. The effect of mutual blending of lines is very large and the true continuum is probably not seen at all in the whole spectral region below $5000 \AA$.

Normally the number of lines decreases with the increasing wave-length and for this reason the red region of the spectrum should be easier to analyse. Unfortunately, high dispersion spectra of $H D 101065$ in the red region are beyond the reach of existing southern telescopes. Only one spectrum of the visual region with a dispersion of $20 \mathrm{~A} / \mathrm{mm}$ has been obtained at Mount Stromlo Observatory, but it is of poor quality. For this reason only limited use of this spectrum is made in the present article. It is hoped that in the near future better spectra will be obtained in the visual range. The present results are based mostly on the best spectrum obtained in the photographic region. Its width is $0.35 \mathrm{~mm}$ and its dispersion $6 \cdot 7 \AA / \mathrm{mm}$. The projected slit width is $28 \mu$.

The effective temperature of $H D 101065$ derived from $B, G, R, I$ colours of the six-colour photometry by $\mathrm{G}$. $\mathrm{E}$. Kron and K. C. Gordon ${ }^{2}$ is $6,350^{\circ} \mathrm{K}$. However, if it is taken into account that the two other colours $U$ and $V$ show the largest known ultra-violet deficiency of 0.4 magnitudes, this temperature should be lowered by about 400 degrees. In the present investigations it has been assumed that the effective temperature of $H D 101065$ is identical with that of the $\operatorname{Sun}\left(5,900^{\circ} \mathrm{K}\right)$.

The parallax of the star is unknown but a fairly high proper motion of $0.054^{\prime \prime}$ per annum, coupled with the low radial velocity of the star $(+10 \mathrm{~km} / \mathrm{sec})$, seems to indicate that the star is not far from the main sequence. It is unlikely that it is a supergiant although it could possibly be a giant of absolute magnitude +1 . However, it is most likely a dwarf on the main sequence and therefore physical parameters of the solar atmosphere used by L. H. Aller ${ }^{5}$ have been assumed in the analysis (the reciprocal temperature $\theta=0.9$ and the logarithm of the electron pressure $\log p_{e}=0 \cdot 9$ ). No estimate of the electron pressure from the spectrum is possible since no element is seen in two stages of ionization.

In view of the fact that $H D 101065$ is an abnormal star, the possibility that some unknown sources of opacity influence its atmosphere cannot be excluded. On the other hand, the agreement of its $B, G, R$ and $I$ colours with those of normal late $F$ type dwarfs suggests that its main source of opacity is the negative hydrogen ion. This has been assumed in the present investigations, since scarcely any other assumptions could properly be justified.

Because of severe mutual blending, very few spectral lines are suitable for an analysis of the spectrum. Only 350 lines of fifteen elements could be used for the abundance analysis. It should be emphasized that almost all equivalent width determinations are only crude estimates which would be disregarded in the analysis of a normal star. However, in view of the importance of $H D 101065$ for the theory of stellar evolution, even an analysis of the chemical composition of its atmosphere based on poor data is certainly better than pure guesswork or complete uncertainty.

In spite of the difficulties in the measurement of the equivalent widths, the individual points on the curve of growth diagrams do not show an obviously larger scatter than on diagrams for normal stars. This may give a certain confidence in the results of the analysis, but does not exclude the possibility of systematic errors in the measurements of the equivalent widths. These errors may be due partly to the error in the continuum (which has been drawn through the highest intensity peaks), partly to the contribution of lines which have not yet been catalogued, and partly to the method used in order to eliminate the effect of blending by neighbouring lines. 